\title{
Non-linear Impedance of a New Sound Absorptive Fibreless Material Title
}

\author{
Fabio Auriemma, Heiki Tiikoja \\ Department of Machinery, Tallinn University of Technology, Tallinn, Estonia \\ e-mail: Fabio.auriemma@ttu.ee
}

\begin{abstract}
The Micro-Grooved Element (MGE) is a novel acoustic solution for noise control aiming to provide high sound absorption coefficient with cost effective technology. In this paper the main characteristics of the MGEs are described, including the geometry and the impedance model. The nonlinear part of the impedance is investigated experimentally by using the 2-port method and modeled with ansatz equations. The different behavior exhibited by the MGEs at different levels of sound excitations is highlighted and general guidelines are included in order to choose the most suitable element according to the operating conditions.
\end{abstract}

Keywords-acoustic materials; absorptive materials; soundproof materials; micro-perforated panels; micro-grooved elements, acoustical impedance, non-linear impedance.

\section{INTRODUCTION}

In applied acoustics, the main problems associated with typical fibrous materials are the non-renewability, the deterioration of the performance over time and the possible separation of small particles that pollute the surrounding media [1]. For this reason the use of fibreless materials, in acoustics, is becoming a common trend in many engineering applications.

Highly absorptive fibreless materials have been applied to room acoustics [2], automotive [3] and aerospace field [4]. In this scenario, one of the first solutions proposed is represented by the micro-perforated elements (MPEs), which have simple structure and well predictable absorption characteristics. Technically, the MPEs are perforated plates with holes and thickness typically in the sub-millimeter range and perforation ratios around $1 \%$. Such characteristics can vary according to the application field and the operating conditions. However, in general these materials exhibit high values of the absorption coefficient in the operating range where the acoustic boundary layer thickness is larger than the aperture radius. The first studies on the MPEs have been presented during the ' 60 s by the Chinese scientist Maa and have been recently revised by the same author [5], [6].

In addition to the micro-circular holes, also microrectangular slits can be utilized in such elements [7]. In both cases a laser beam treatment is used to manufacture the apertures. Accordingly, the traditional MPEs are often considered too expensive for commercial applications. For this reason several fibreless solutions have been recently proposed, aiming to exploit the same physical principles while reducing the production costs [8], [9], [10], [11].

An important aspect connected to the acoustic performance of the perforated elements is represented by the non-linear part of the acoustic impedance. This term is here utilized to describe the non-linear relationship between the acoustic pressure amplitude and the particle velocity taking place when the sound excitation exceeds a certain level. The non-linearities have been object of several studies since the 60 s' due to the strong effect on the behavior of the perforated elements [12], [13], [14].

In this paper, the novel MGEs are studied experimentally and theoretically with special attention to non-linear effects. The acoustic impedance exhibited by MGEs provided with different internal geometries is in focus. The interactions between adjacent channels have been accounted too. A semi empirical approach has been followed in order to model the acoustic impedance by means of ansatz equations. Finally, the effects of the nonlinear part of the impedance on the absorption coefficient are highlighted.

\section{THE ACOUSTICS OF MiCRO-GROOVED ElEMENTS}

The MGEs have been first described in [11] and here a brief summary is reported. A MGE is typically composed of at least two overlying layers presenting macroscopic slots and a number of micro-grooves at least on one of the contact surfaces (see Fig.1a and Fig. 1b). In contrast with the typical MPEs, the MGEs use the grooves, instead of the holes, which the air is forced to pass through. Once the constituting layers are mated, such groves form a number of micro-ducts. In this way the sound waves enter the element via the slots of the first layer - the inlet -, pass through the micro-ducts where dominant energy dissipation is expected to occur, and finally exit from the slots of the second layer - the outlet -. This concept allows replacing laser perforation process (typically required for small series custom designed MPEs) with cost effective and considerably less time-consuming burr removal process. The two constituting layers can be coupled to each other by welding, riveting or clamping.

A variety of different MGEs have been tested in this work. The samples differ for the porosity $\sigma$, the depth $d$ of the channels, the width $w$ of the channels, the length $t$ of the channels, and the channel configuration. All the samples examined here have the width of the slots on the outlet layer $g=4 \mathrm{~mm}$, which has been seen to be a preferable choice [11]. The Table I summarizes the ranges of variability of the previously mentioned parameters in the samples examined. Only two values of the width $w$ have been considered. MGEs with $w=21.15 \mathrm{~mm}$ are of the type represented in Figure 1a, i.e. the micro-channels are spaced apart (conventionally $i=\infty$ ) and mutual interactions might occur in 
specific conditions only between two facing micro-channels (e.g. channels $c_{1}$ and $c_{2}$ in Fig. 1a). See [11] for details.

MGEs with $w=2.15$ are of the type represented in Fig. 1b, i.e. the micro-channels are placed close to each other (parameter $i=3 \mathrm{~mm}$ ). In this case mutual interactions always take place between two adjacent micro-channels (e.g. channels $c_{1}$ and $c_{3}$ in Fig. 1a). Since the experiments have been carried out by using different sound excitation levels, the Table 1 also includes the values of sound pressure level (SPL) measured within the 2-port test rig during the experiments. An accurate description of the test rig utilized in these experiments can be found in [11].

\section{IMPEDANCE CHARACTERIZATION}

\section{A. Transfer Impedance}

The transfer impedance $\left(Z_{p}\right)$ is believably the most suitable parameter for the acoustic characterization of the performance of thin acoustic materials. It represents the ratio between acoustic pressure drop $\Delta p$ through the material and the normal particle velocity $u$, which is regarded as constant for thin panels (see the Figure 1a).

The transfer impedance of a panel $\left(Z_{p}\right)$ is usually related to the air impedance $(Z=\rho c)$, where $\rho$ is the air density and $c$ is the speed of sound, resulting in the so-called normalized impedance $\left(z_{p}=Z_{p} / Z\right)$.

The real part of the transfer impedance $(r)$ is typically referred to as resistive part or transfer resistance. It is related to the viscous losses taking place in the apertures. The imaginary part of the transfer impedance $(\chi)$ is typically referred to as reactive part or transfer reactance. It is related to the inertia of the air plugs of the apertures. Under the assumption of constant particle velocity throughout the MGE, the normalized transfer impedances of the inlet layer $\left(Z_{\text {in }}\right)$, of the micro-channels $\left(Z_{\text {micro }}\right)$ and of the outlet layer $\left(Z_{\text {out }}\right)$ are series impedances (see Figure 1b). Moreover, due to the presence of large apertures characterizing the inlet layer, the impedance of this layer is negligible. Thus, the normalized transfer impedance of the MGE can be written as: $Z_{M G E}=Z_{\text {micro }}+Z_{\text {out }}$. The resistive part of $Z_{\text {out }}$ is also typically negligible. Once the reactive part of $Z_{\text {out }}$ has been measured, $Z_{\text {micro }}$ can be extracted from the measured ZMGE. The experimental procedure is detailed in [11].

\section{B. Internal and External Parts}

Internal and external parts of the transfer impedance are respectively related to the phenomena occurring within the micro-channels and the end corrections of the microchannels. The end corrections are corrective terms of the inner impedance which are used to include the extra viscous losses and air inertia contributions at the endings of the air plugs.

In [11] the internal and external parts of micro-channels in MGEs are measured and modeled by means of ansatz equations. For readability reasons the main results are only summarized here. $Z_{\text {out-i }}$, i.e., the internal transfer impedance of the outlet layer, and $Z_{\text {micro-i }}$, the internal transfer impedance of the micro-channels, can be modeled by using the impedance formulation for slip shaped apertures.
TABLE I. GEOMETRICAL PARAMETERS OF THE MGES TESTED AND SOUND EXCITATION LEVELS USED IN THE EXPERIMENTS.

\begin{tabular}{|l|c|c|c|}
\hline \multirow{2}{*}{ Parameter } & \multicolumn{3}{|c|}{ Range } \\
\cline { 2 - 4 } & $\begin{array}{c}\text { Non- } \\
\text { dimensional }\end{array}$ & $d B$ & $\mathrm{~mm}$ \\
\hline$\sigma$ & {$[0.008,0.05]$} & & {$[0.1,0.5]$} \\
\hline $\mathrm{d}$ & & & $2.15,21.15$ \\
\hline $\mathrm{w}$ & & & {$[0.75,2.75]$} \\
\hline $\mathrm{t}$ & & & $3, \infty$ \\
\hline $\mathrm{i}$ & & {$[110,145]$} & \\
\hline $\mathrm{SPL}$ & & & \\
\hline
\end{tabular}

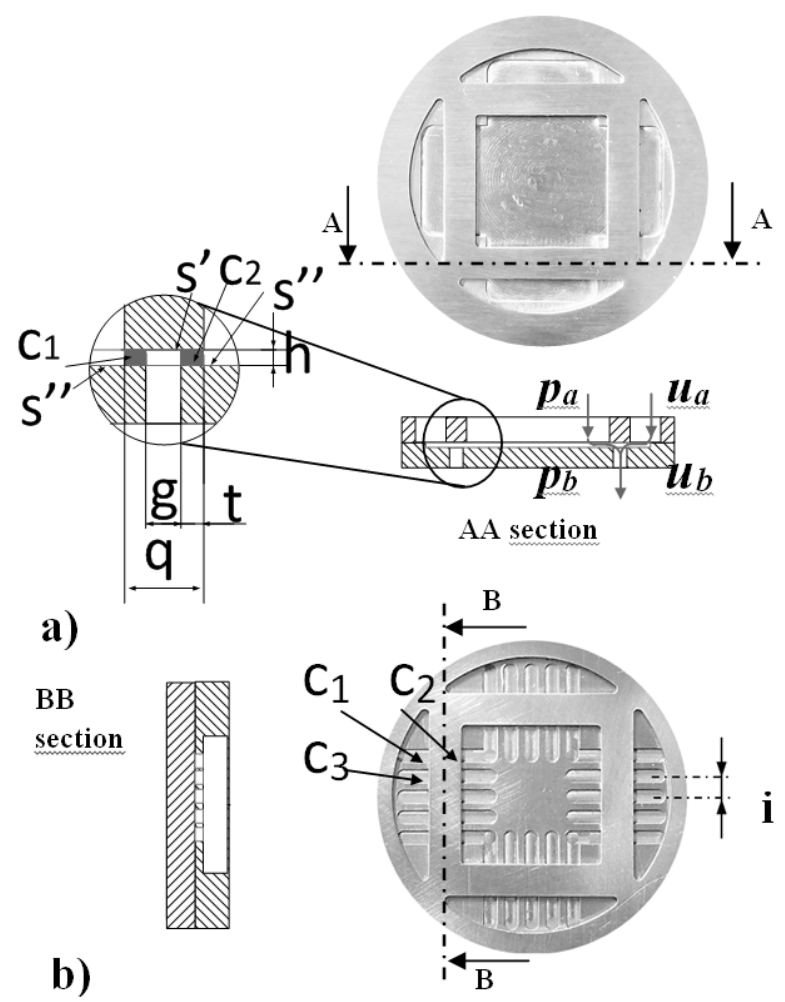

Figure 1. Micro-grooved elements. a) Configuration with 8 wide noninteracting micro-channels. b) Configurations with 40 narrow interacting micro-channels.

Contrariwise, the formulation typically utilized to describe the external impedance of plates with slit shaped aperture must be handled carefully in order to be used for MGEs. This formulation can be used to model $Z_{\text {out }- \text {, }}$ i.e., the external transfer impedance of the outlet layer, and the end resistance values of the MPE with non-interacting microchannels (as in Figure 1a).

However, for samples with interacting adjacent microchannels (as in Figure 1b) a corrective factor of 1.5 is required to model the end resistance. Moreover, the reactive end correction exhibited by the micro-channels included in 
the MGEs, $\chi_{\text {micro-e }}$, is always greater than the reactive end correction exhibited by perforated plates, $\chi_{\text {slite }}$, with the same geometry. This is due to the presence of the surfaces $s$, and $s$ " which results in an increased inertia of the air plug contained in the micro-channel $c^{\prime}$ (see Figure 1a).

\section{NON-LiNEAR EFFECTS IN MGES}

Non-linear effects occur in perforated plates and MGEs when the sound pressure level of the sound source is increased above a certain threshold. In this case a third contribution (non-linear part) appears in the transfer impedance resulting in a non-linear (quadratic) relationship between the acoustic pressure amplitude and the particle velocity. As a consequence, the resistance increases and the reactance decreases.

The non-linear part of the transfer impedance can be measured by subtracting the linear and external part from the impedance of the micro-channels. Thus, the resistive part can be obtained as $r_{\text {micro-NL }}=r_{\text {micro }}-r_{\text {micro-i }}-r_{\text {micro-e }}$. Moreover, the reactive part is a function of the external reactance $\chi_{\text {micro- }}$ $e$. For this reason the term $\chi_{\text {micro-NL }}=\chi_{\text {micro }}-\chi_{\text {micro- } i}$ already includes $\chi_{\text {micro-e }}$.

The following formulations regarding the non-linear transfer impedance of slit shaped apertures can be found in literature:

$$
\begin{gathered}
r_{\text {slit }-N L}=\frac{\left|u_{h}\right|}{\sigma c} \\
\chi_{\text {slit }-N L}=\chi_{\text {slit }-e}\left(1+\frac{\left|u_{h}\right|}{\sigma c}\right)^{-1}
\end{gathered}
$$

In the Eq. 1 and 2, uh is the peak particle velocity within the apertures. These equations are valid for pure tone excitation. The Equations 1 and 2 must be modified in order to describe the non-linear behavior of the micro-channels in MGEs.

In [11] the equations for non-linear impedance of microchannels have been carried out for wide non interacting micro-channels (as in Figure 1b). The following formulation has been found for white noise excitation:

$$
\begin{gathered}
r_{\text {slit }-N L}=\frac{F\left|u_{h}\right|}{1000 \sigma c} \\
\chi_{\text {slit }-N L}=\chi_{\text {slit-e }}\left(1+\frac{F\left|u_{h}\right|}{1000 \sigma c}\right)^{-1}
\end{gathered}
$$

Where $F$ is a factor depending on the geometry of the micro-channels:

$$
F=\frac{n_{1} r m s_{u-\text { total }}+n_{2}}{r m s_{u-\text { total }}+n_{3}}
$$

$r m s_{u-t o t a l}$ is the $r m s$ value of the particle velocities within the apertures across when the white noise is utilized. Moreover,

$$
\left\{\begin{array}{l}
n_{1} \\
n_{2} \\
n_{3}
\end{array}\right\}=\left[\begin{array}{ccc}
+7.0 E-02 & -8.4 E+03 & +3.1 \\
-9.6 E+07 & +6.8 E+04 & -12 \\
-5.4 E+07 & +3.4 E+04 & -5.1
\end{array}\right]\left\{\begin{array}{l}
d^{2} \\
d \\
1
\end{array}\right\}
$$

The Eq.14 shows that the non-linear part of the impedance has a strong dependency from the depth $d$ of the channels and it is independent on the length $t$ of the microchannels.

Here, the effect of the mutual interaction among adjacent channels (as for the configuration in Figure 1a) is investigated too. However, it must be pointed out that all the samples utilized to this aim have the same distance $i=3 \mathrm{~mm}$ among adjacent micro-channels. In this case, other two corrective coefficients $F_{r e s}^{\prime}$ and $F_{\text {rea }}^{\prime}$ are introduced to describe the non-linear part of the impedance, resulting in $r^{\prime}{ }_{\text {slit }}=F^{\prime}{ }_{\text {res }} r_{\text {slit-NL }}$ and $\chi_{\text {slit }-N L}^{\prime}=F^{\prime}{ }_{\text {rea }} \chi_{\text {slit-NL }}$.

The Figure 2 shows that $F^{\prime}$ res is essentially a function of the porosity, and it increases as the porosity decreases. For this reason, the viscous losses taking place within the apertures, related to the particle velocity therein, are highly increased. However, a different behavior is exhibited by $F^{\prime}{ }_{\text {rea }}$, as shown in the Figure 3 . In fact, this parameter mainly depends on the length $t$ of the micro-channels and it is reasonable to think that, at increasing $t$, the inertia of the air plug increases too.

The results presented in the Figure 2 and 3 have been interpolated by using, in the first case, rational functions of $r m s_{u-t o t a l}$ with linear numerator and denominator and, in the second case, by using linear functions of $r m s_{u-t o t a l}$. The dependence of $F^{\prime}{ }_{\text {res }}$, on porosity, and of $F^{\prime}{ }_{\text {rea }}$, on microchannel length, is quadratic in both cases.

As results, the following equations have been found:

$$
\begin{gathered}
F_{r e s}^{\prime}=\frac{3.7 r m s_{u-\text { total }}-16.4}{r m s_{u-\text { total }}-4.3}+1.7 \sigma^{2}-3.2 \sigma-0.15 \\
F_{r e a}^{\prime}=\left(4.1 E+03 t^{2}-14 t+1.6 E-02\right) r m s_{u-\text { total }}+9.6 E-01 \\
\text { V. ABSORPTIVE COEFFICIENT }
\end{gathered}
$$

\section{ABSORPTIVE COEFFICIENT}

The absorptive coefficients of three different MGEs, referred to as Samples A, B and C, backed by a $25 \mathrm{~mm}$ length cavity, have been measured at two different levels of sound excitation. The results are plotted in the Figure 4 and 5. The Sample A is made of 40 adjacent interacting small micro-channels (of the type in Figure 1b), with $1.96 \%$ porosity. The samples $\mathrm{B}$ and $\mathrm{C}$ are provided with 8 wide micro-channels (of the type in Figure 1a), with the same width $(21.15 \mathrm{~mm})$, and different depth. The porosities are $1.35 \%$ and $3.62 \%$ for Samples B and C respectively.

The ideal condition of frequency constant unitary absorption coefficient is given by a frequency constant unitary normalized transfer resistance and a frequency constant null normalized transfer reactance.

However, although the condition on the resistance can be achieved in MPEs and MGEs, typically the reactance is a linearly growing function of the frequency, resulting in a hump shaped absorption coefficient. Despite of the similar porosity of the Samples A and B, the narrower absorption band of the Sample A at $110 \mathrm{~dB}$ is related to the fact that, for mutually interacting micro-channels, the reactance increases markedly and reaches high values ( 4) at $3000 \mathrm{~Hz}$. 


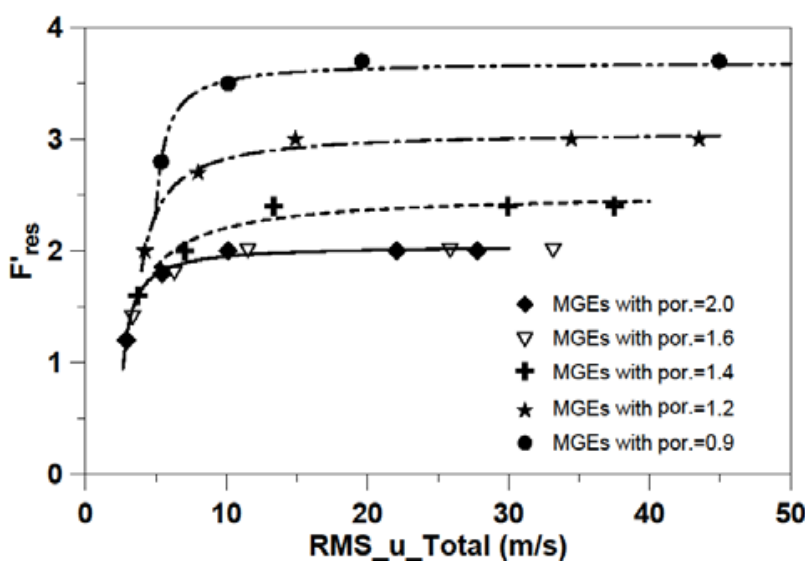

Figure 2. F' ${ }^{\prime}$ res coefficient as a function of particle velocity and element porosity.

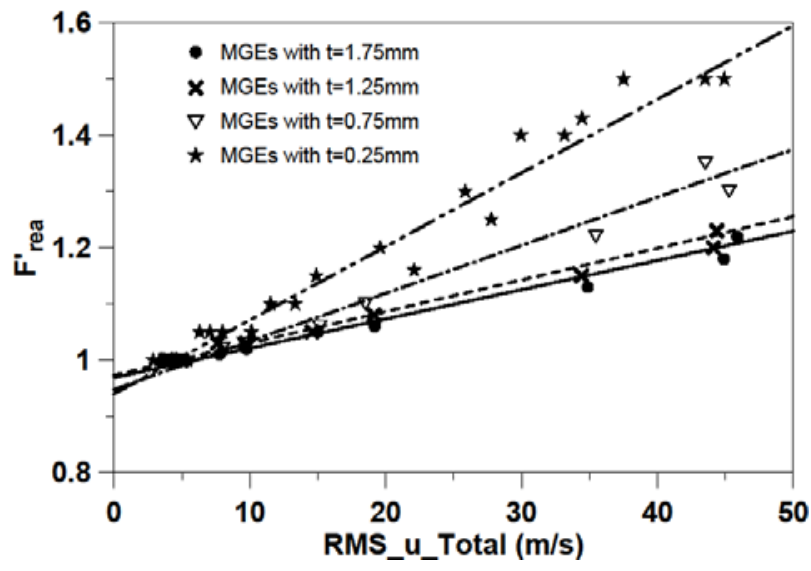

Figure 3. F'rea coefficient as a function of particle velocity and length of the micro-channels.

It is noticeable that these samples respond differently to the variation of sound excitation. The behavior of the Sample B at $110 \mathrm{~dB}$ is optimal, with a wide hump and a peak around 1 , because the normalized transfer resistance is unitary and the normalized transfer reactance is a slowly increasing function of the frequency. The drop of absorption coefficient at $140 \mathrm{~dB}$ is related to the increase of the normalized resistance above the unit, in the situation where the particle velocity rises. However, the reduction of the normalized reactance, which happens when the sound excitation increases, has shown a positive effect of flattening the curves of the absorption coefficient (see Figure 5).

The MGE Sample C has been designed for high sound excitation levels because of the higher porosity and bigger depth of the micro-channels. From Figure 4 and 5, it can clearly be seen that, at low excitation levels, the absorption coefficient is relatively small due to the very low resistance of the element. Contrariwise, at high sound excitation levels, the acoustic performance is excellent in terms of the peak value and the shape of the absorption coefficient curve.

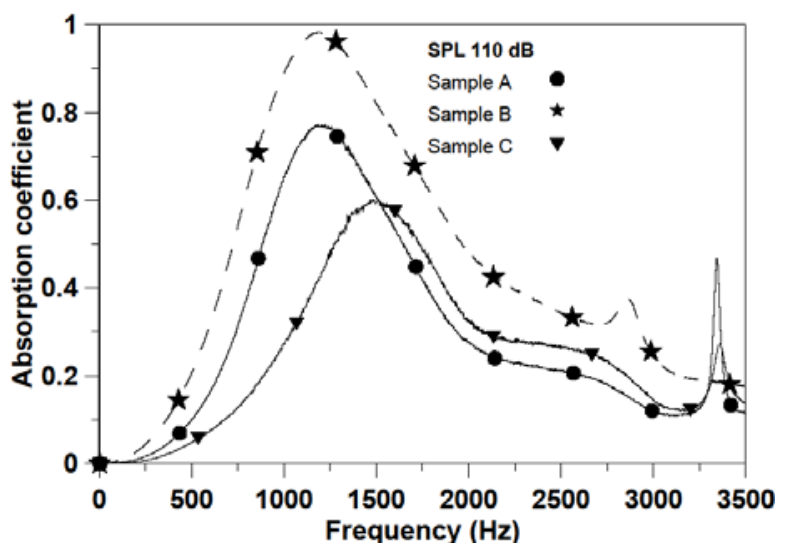

Figure 4. Absorptive coefficients of Samples A, B, C at $110 \mathrm{~dB}$.

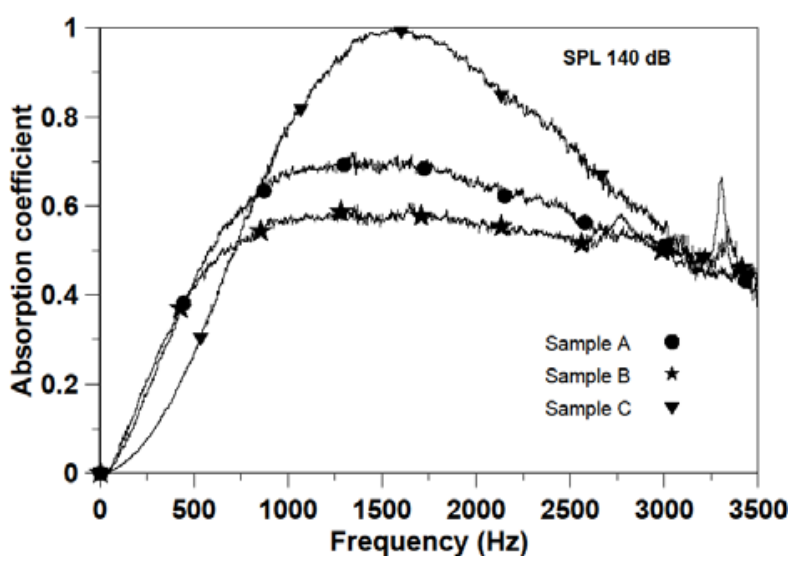

Figure 5. Absorptive coefficients of Samples A, B, C at $140 \mathrm{~dB}$.

In fact, in this condition the normalized transfer resistance reaches the unitary value, which allows the absorption coefficient to be $\sim 1$ at a certain frequency. Moreover, the non-linear effects induce reduction of the reactance resulting in a flattened absorption curve, whose values are higher than 0.6 for almost $2 \mathrm{kHz}$ frequency range.

\section{CONCLUSIONS}

In this paper, the acoustical transfer impedance of MGEs has been studied with special attention to the effects of the mutual interactions among adjacent micro-channels. Moreover, the non-linear part of the transfer impedance has been widely investigated too.

The internal and external part of the transfer impedance of MGEs can be written as functions of the internal and external part of the transfer impedance of plates provided with rectangular slits. The resistive part of the end correction increases in case of MGEs provided with small interacting adjacent micro-channels. The reactive external part of the 
micro-channels in MGEs is always greater than the reactive external part of slit shaped apertures - with the same geometry - in plates.

The non-linear effects have been expressed as a function of the particle velocity within the micro-channels and the geometry of the micro-channels. In case of MGEs provided with small mutually interacting micro-channels, the reactive non-linear part of the transfer impedance is a quadratic function of the sample porosity. The reactive non-linear part is a quadratic function of the length of the micro-channels.

The non-linear part of the transfer impedance significantly affects the behavior of the MGE at the extent that different MGEs are recommended to be used at different sound excitation levels. Usually MGEs with small interactive adjacent micro-channels result in narrow absorption curves. Wide channels and porosities $\sim 1 \%$ are preferable when non-linear effects are negligible, i.e. low levels of sound excitation. Wide channels and higher porosities (up to $\sim 4 \%$ ) are desirable when non-linear effects are predominant, i.e. high levels of sound excitation.

\section{REFERENCES}

[1] D. Herrin, J. Liu, Sound and vibration magazine, Properties and applications of microperforated panels, 2011.

[2] T.J. Cox, P. D'Antonio, Acoustic absorbers and diffusers, Taylor and Francis group, London and New York, ISBN 0-203-89305-0, 2009.
[3] F. Auriemma, H. Rämmal, J. Lavrentjev, JSAE 20139001, Application of micro-grooved elements to small engine silencer, SETC Japan, 2013.

[4] G. W. Bielak, J. W. Premo, A. S. Hersh, Tech. rep., NASA, Advanced turbofan duct liner concepts, Langley Research Center Hampton, Virginia, USA, 1999.

[5] D. Y. Maa, Proceedings of Internoise 94, Microperforated panel at high sound intensity, Yokohama, Japan, 1994.

[6] D. Y. Maa, Journal of Acoustic Society of America, 104(5), Potential of microperforated panel absorber, 1998.

[7] J. F. Allard, N. Atalla, Propagation of sound in porous media Modeling sound absorbing materials, John Wiley \& Sons, Ltd publication ISBN: 978-0-470-746615-0, 2009.

[8] Knipstein D. M., Tech. Rep. patent number EP0876539B1, Sound absorbing element and procedure for manufacture, European patent Office

[9] Randeberg R. T., 2000, Perforated panel absorbers with viscous energy dissipation enhanced design, Ph.D. thesis, Trondheim, Norway.

[10] Pfretzschner J., Fernandez A., 2006, Applied Acoustics 67, Microperforated insertion units.

[11] Auriemma F., Rämmal H., Lavrentjev J., 2013, "SAE International Journal of Materials and Manufacturing 3 (2013) 599-610, A novel solution for noise control, doi: 10.4271/2013-01-1941.

[12] Ingard U., 1967, Journal of Acoustic Society of America, Vol.43, Nonlinear attenuation of Sound in a Duct.

[13] Ingard U., 1967, Journal of Acoustic Society of America, Vol.42, Acoustic Nonlinearity of an orifice.

[14] Tayong R., Leclaire P., 2011, Applied Acoustics, Experimental investigation of perforations interactions effects under high sound pressure levels, DOI: 10.1016/j.apacoust.2011.04.011. 